Communications in Physics, Vol. 24, No. 4 (2014), pp. 333-342

DOI: $10.15625 / 0868-3166 / 24 / 4 / 3268$

\title{
OPTICAL PHONON MODES AND ELECTRON-OPTICAL PHONON INTERACTION IN CORE-SHELL SEMICONDUCTOR QUANTUM WIRES
}

\author{
NGUYEN NHU DAT \\ Institute of Physics, Vietnam Academy of Science and Technology \\ and \\ Duy Tan University, Da Nang, Vietnam \\ LE THANH HAI \\ Hanoi University of Civil Engineering, Vietnam \\ E-mail: nndat@iop.vast.ac.vn
}

Received 19 June 2014

Accepted for publication 12 November 2014

\begin{abstract}
Within the framework of the macroscopic dielectric continuum model the longitudinal optical (LO) phonon modes are derived for a cylindrical semiconductor quantum wire made of semiconductor 1 (well material) embedded in another finite semiconductor 2 (barrier material). The phonon states of modes are given by solving the generalized Born-Huang equation. It is shown that there may exist four types of longitudinal optical phonon modes according to the concrete materials forming the wire. The dispersion equations for phonon frequencies with wave-vector components parallel to the wire are obtained. After having quantized the phonon field we derive the Fröhlich Hamiltonian describing the electron-LO-phonon interaction. The influence of the thickness of the barrier layer as well as the thin metallic shell on the phonon frequencies and their interaction with electrons is studied.
\end{abstract}

Keywords: core-shell wire, thickness, phonon, dispersion.

\section{INTRODUCTION}

The progress in semiconductor nanotechnology has made it possible to fabricate quasione-dimensional wire structures of different semiconductor materials. These semiconductor wire structures have lateral dimension small relative to the electron thermal de Broglie wavelength and, therefore, exhibit a number of new, so-called quantum size effects which make the structures attract much attention due to their potential technological application [1-9].

The electron-phonon interaction in semiconducting low-dimensional systems in general, and in one-dimensional quantum wires in particular, is of great importance since it is one of the main mechanisms that govern carrier relaxation in transport phenomena. In the past, several workers [10-15] had carried out studies of the electron interaction with longitudinal optical (LO) phonons in quantum wires considering the modification of the electron energy spectrum and wave function due to their space localization but assuming the bulk forms for LO phonon modes. 
OPTICAL PHONON MODES AND ELECTRON-OPTICAL PHONON INTERACTION ...

However, it was shown that the reduced sized scale and dimensionality have great influence leading to phonon confinement and changes in phonon modes [16-19]. These changes become an essential part of the description of electron-phonon interaction and affect strongly the transport properties of electrons in quantum wires.

There exist various theoretical proposals in the literature to treat longitudinal optical vibrations in semiconductor nanostructures. Microscopic models [20,21] have been developed to describe LO phonons in low-dimensional systems which required the labored numerical calculation. Macroscopic theories have been successfully employed to investigate optical phonons giving a proper analytical description for the phonon confinement. These theories are based on continuum models such as the dielectric continuum (DC) model [22-29], the hydrodynamic (HD) model [30-32], and the third, so-called hybrid model [33-37]. The DC model requires the fulfillment of the electrostatic conditions at the boundaries, whilst the optical phonon modes in the HD model are determined by the mechanical boundary conditions. It is clear that the DC model failed to satisfy mechanical boundary conditions and conversely, the HD approach violated the electromagnetism. The DC model anticipates the existence of the two kinds of optical phonon modes, confined modes and interface modes, for example, in GaAs/AlAs heterostructures. The HD model agrees on the form of the interface modes but predicts different symmetry of the electrostatic potential of the confined modes. The hybrid model suggests that the two above outlined approaches should be combined in a proper way, taking into account simultaneously both mechanical and electrostatic boundary conditions. Among these models, the DC approach had been widely employed due to its simplicity.

To our knowledge, most of works dealing with optical phonons and their interaction with electrons in a quasi one-dimensional semiconductor wire studied the system in the form of either a free-standing quantum wire or being enclosed in another infinite material. In the present work, we consider a quantum wire formed by polar semiconductor material 1 of the shape of a cylinder of radius $R_{1}$ which is embedded in barrier polar semiconductor 2 of the form of a hollow cylinder with inner radius $R_{1}$ and outer radius $R_{2}$. We concentrate on the effects of the finite thickness of the barrier. We will, therefore, apply the DC approach to develop the eigenfunctions of LO phonons on the basis of the generalized Born-Huang equation and investigate the influence of the thickness of the barrier layer on the LO phonon dispersion relation and the electrostatic potential related to LO vibrations. Furthermore, we also consider the quantum wires having an outer thin metal shell in addition and show that the presence of the metal shell affects considerably not only the frequencies of LO phonons but the connected electric potential as well.

\section{LO-PHONON MODES}

Let us consider a more realistic model of a semiconductor circular wire, made of a polar material (denoted by 1 ), of radius $R_{1}$ surrounded by another material (denoted by 2 ) filling up a hollow cylinder with inner radius $R_{1}$ and outer radius $R_{2}$. The outmost environment is vacuum. We assume the wire is of large length $L$, take the $z$ axis along the wire, denote the radial distance as $r$ and the azimuthal angle as $\varphi$. We work in the frame of the dielectric continuum model to derive the various phonon modes and their interaction Hamiltonian with electrons for the quantum wire.

We denote by $\vec{u}(\vec{x}, t)$ the relative atomic displacement of the two atoms of a unit cell at a lattice node $\vec{x}$ which is considered to be continuously varying. Assuming the medium to be 
elastically isotropic, the displacement $\vec{u}$ is coupled to the electric field $\vec{E}$ by the equation

$$
\ddot{\vec{u}}(\vec{x}, t)=-\omega_{j T}^{2} \vec{u}(\vec{x}, t)-v_{j L}^{2} \vec{\nabla}(\vec{\nabla} \cdot \vec{u}(\vec{x}, t))-v_{j T}^{2} \vec{\nabla} \times(\vec{\nabla} \times \vec{u}(\vec{x}, t))+\frac{\varepsilon_{j \infty} e_{j}^{*}}{M_{j}} \vec{E}(\vec{x}, t) .
$$

where $\omega_{j T}$ and $v_{j T}, v_{j L}$ are the limiting bulk transverse optical frequency and certain velocities, respectively, of material $j, j=1,2$, for $\vec{x}$ in material $j$. This is the generalized Born-Huang equation in which parameters $v_{j L}$ and $v_{j T}$ are introduced to account for the spatial dispersion of the vibrations. The quantity $e_{j}^{*}$ is referred to as a dynamic, or Callan, effective charge,

$$
e_{j}^{*}=\omega_{j L} \sqrt{\frac{1}{4 \pi} \frac{M_{j}}{N_{j}}\left(\frac{1}{\varepsilon_{j \infty}}-\frac{1}{\varepsilon_{j 0}}\right)} .
$$

Here $M_{j}$ and $N_{j}$ mean the reduced mass of the two atoms of a unit cell and the density of unit cells of material $j$, respectively. $\omega_{j L}$ is the bulk longitudinal optical frequency related to $\omega_{j T}$ via the Lyddane-Sachs-Teller relation. $\varepsilon_{j \infty}$ and $\varepsilon_{j 0}$ are the high-frequency and static dielectric constant of material $j$. We use $\varepsilon_{0}$ to denote the dielectric constant of vacuum.

Equation (1) is supplemented by the familiar source-free Maxwell equations, one of which is the equation for the electric displacement $\vec{D} \equiv \vec{E}+4 \pi \vec{P}$,

$$
\operatorname{div} \vec{D}=0
$$

and the equation connecting the polarization field $\vec{P}$ with the electric field $\vec{E}$ and the mechanical displacement field $\vec{u}$,

$$
\vec{P}(\vec{x}, t)=\omega_{j T}\left[M_{j} N_{j}\left(\varepsilon_{j 0}-\varepsilon_{j \infty}\right) /(4 \pi)\right]^{1 / 2} \vec{u}(\vec{x}, t)+\left[\left(\varepsilon_{j \infty}-1\right) /(4 \pi)\right] \vec{E}(\vec{x}, t) .
$$

For LO vibrations the electric displacement field $\vec{D}$ vanishes, $\vec{D}=0$. Thus, after simple mathematical manipulation, the equation for the time Fourier transform $\vec{u}(\vec{x})$ of the displacement field $\vec{u}(\vec{x}, t)$ can be written as

$$
\left[\omega_{j L}^{2}-\omega^{2}\right] \vec{u}(\vec{x})+v_{j L}^{2} \Delta \vec{u}(\vec{x})=0
$$

We adopt the Born-von Karman boundary condition in the $z$ direction, i.e., we demand $\vec{u}(\vec{x})=\vec{u}\left(\vec{x}+L \vec{e}_{z}\right)$, where $\vec{e}_{z}$ means a unit vector parallel to $z$. Exploiting the translational and rotational symmetry of the wire we can write the eigenfunctions of Eq.(5) in the form

$$
\vec{u}_{v m q}(\vec{x})=\vec{u}_{v m q}(r) e^{i(m \varphi+q z)} .
$$

Here the wave number $q$ and the integer number $m$ characterize irreducible representations of the corresponding symmetry groups. The index $v$ corresponds different phonon types and branches for given $q$ and $m$ as shown in the forthcoming.

Since the displacement $\vec{u}_{v m q}(\vec{x})$ conforms to the equation $\vec{\nabla} \times \vec{u}_{v m q}(\vec{x})=0$ for longitudinal vibrations, it may be expressed as a gradient of a scalar function

$$
\vec{u}_{v m q}(\vec{x})=\vec{\nabla}\left\{\psi_{v m q}(r) e^{i(m \varphi+q z)}\right\}
$$

which in turn, from Eq. (5), obeys the following equation

$$
\left[\frac{d^{2}}{d r^{2}}+\frac{1}{r} \frac{d}{d r}+\left(\frac{\omega_{j L}^{2}-\omega^{2}}{v_{j L}^{2}}-q^{2}-\frac{m^{2}}{r^{2}}\right)\right] \psi_{v m q}(r)=0
$$


with $j=1$ or 2 for $r<R_{1}$ or $R_{1}<r<R_{2}$, respectively. The solutions of Eq. (8) involve Bessel and modified Bessel functions of various kinds depending on whether the wave number

$$
k_{j}=\sqrt{\frac{\omega_{j L}^{2}-\omega^{2}}{v_{j L}^{2}}-q^{2}}
$$

is real or imaginary. For imaginary $k_{j}$ we put $k_{j}=i \kappa_{j}$ with $\kappa_{j}=\left|k_{j}\right|$. It is straightforward to show that solutions can be written in the form

$$
\psi_{v m q}(r)= \begin{cases}A F_{m}\left(k_{1} r\right), & r<R_{1} \\ B F_{m}\left(k_{2} r\right)+C G_{m}\left(k_{2} r\right), & R_{1}<r<R_{2}\end{cases}
$$

where constants $A, B$ and $C$ are determined from boundary conditions.

If $k_{j}$ is real, $F_{m}\left(k_{j} r\right)$ is a Bessel function of the first kind $J_{m}\left(k_{j} r\right)$ of order $m$, and $G_{m}\left(k_{j} r\right)$ is a Bessel function of the second kind (Neumann function) $Y_{m}\left(k_{j} r\right)$. When $k_{j}$ is imaginary the function $F_{m}\left(k_{j} r\right)$ is an order- $m$ modified Bessel function of the first kind (Infeld function) $I_{m}\left(\kappa_{j} r\right)$, and $G_{m}\left(k_{j} r\right)$ a modified Bessel function of the second kind (MacDonald function) $K_{m}\left(\kappa_{j} r\right)$. The definitions and conventions of Bessel functions are taken from [38]. Applying Eq. (7) one gets the corresponding displacement eigenfield

$$
\vec{u}_{v m q}(\vec{x})=e^{i(m \varphi+q z)}\left\{\begin{array}{cc}
A\left[k_{1} F_{m}^{\prime}\left(k_{1} r\right) \vec{e}_{r}+\frac{i m}{r} F_{m}\left(k_{1} r\right) \vec{e}_{\varphi}+i q F_{m}\left(k_{1} r\right) \vec{e}_{z}\right], & r<R_{1} \\
k_{2}\left[B F_{m}^{\prime}\left(k_{2} r\right)+C G_{m}^{\prime}\left(k_{2} r\right)\right] \vec{e}_{r}+\frac{i m}{r}\left[B F_{m}\left(k_{2} r\right)+C G_{m}\left(k_{2} r\right)\right] \vec{e}_{\varphi} \\
+i q\left[B F_{m}\left(k_{2} r\right)+C G_{m}\left(k_{2} r\right)\right] \vec{e}_{z}, \quad R_{1}<r<R_{2} .
\end{array}\right.
$$

The prime at a function means the derivative with respect to the argument of the function. $\vec{e}_{r}, \vec{e}_{\varphi}$ and $\vec{e}_{z}$ are unit cylindrical vectors. The classification of the longitudinal optical phonon modes is given in Table 1. The electric field connected with the longitudinal optical vibrations is given by

Table 1. LO-phonon modes of a cylindrical quantum wire

\begin{tabular}{|c|c|c|c|}
\hline \hline \multirow{2}{*}{ Type of modes } & \multirow{2}{*}{$k_{j}$} & \multicolumn{2}{|c|}{$\psi_{v m q}(r)$} \\
\cline { 2 - 4 } & & $r<R_{1}$ & $R_{1}<r<R_{2}$ \\
\hline 1 & real $k_{1}$, real $k_{2}$ & $A J_{m}\left(k_{1} r\right)$ & $B J_{m}\left(k_{2} r\right)+C Y_{m}\left(k_{2} r\right)$ \\
2 & real $k_{1}$, imaginary $k_{2}$ & $A J_{m}\left(k_{1} r\right)$ & $B I_{m}\left(\kappa_{2} r\right)+C K_{m}\left(\kappa_{2} r\right)$ \\
3 & imaginary $k_{1}$, real $k_{2}$ & $A I_{m}\left(\kappa_{1} r\right)$ & $B J_{m}\left(k_{2} r\right)+C Y_{m}\left(k_{2} r\right)$ \\
4 & imaginary $k_{1}$, imaginary $k_{2}$ & $A I_{m}\left(\kappa_{1} r\right)$ & $B I_{m}\left(\kappa_{2} r\right)+C K_{m}\left(\kappa_{2} r\right)$ \\
\hline
\end{tabular}

$$
\vec{E}(\vec{x})=-4 \pi N_{j} e_{j}^{*} \vec{u}(\vec{x}) .
$$

In order to obtain the mode frequencies we need specify the matching boundary conditions at the interfaces between different media. We adopt the conventional electrostatic boundary conditions which can be stated as follows

- Continuity at the interface of the normal component $D_{n}$ of the electric displacement $\vec{D}$. 
- Continuity at the interface of the tangential component $E_{t}$ of the electric field $\vec{E}$. In case of existence of an outer metallic shell, the tangential part of the electric field vanishes at the metal shell.

Applying these above boundary conditions we are able to express two coefficients among $A, B$ and $C$ in Eq. (11) in terms of the third one which in turn will be determined from normalization. We also deduce the following dispersion relations for phonons of different types in considered quantum wires

- Phonons of type 1

$$
\begin{array}{r}
\varepsilon_{1 \infty} k_{1} J_{m}^{\prime}\left(k_{1} R_{1}\right)\left\{\varepsilon_{0} q K_{m}^{\prime}\left(q R_{2}\right)\left[J_{m}\left(k_{2} R_{1}\right) Y_{m}\left(k_{2} R_{2}\right)-Y_{m}\left(k_{2} R_{1}\right) J_{m}\left(k_{2} R_{2}\right)\right]\right. \\
\left.+\varepsilon_{2 \infty} k_{2} K_{m}\left(q R_{2}\right)\left[Y_{m}\left(k_{2} R_{1}\right) J_{m}^{\prime}\left(k_{2} R_{2}\right)-J_{m}\left(k_{2} R_{1}\right) Y_{m}^{\prime}\left(k_{2} R_{2}\right)\right]\right\} \\
+\varepsilon_{2 \infty} k_{2} J_{m}\left(k_{1} R_{1}\right)\left\{\varepsilon_{0} q K_{m}^{\prime}\left(q R_{2}\right)\left[Y_{m}^{\prime}\left(k_{2} R_{1}\right) J_{m}\left(k_{2} R_{2}\right)-J_{m}^{\prime}\left(k_{2} R_{1}\right) Y_{m}\left(k_{2} R_{2}\right)\right]\right. \\
\left.+\varepsilon_{2 \infty} k_{2} K_{m}\left(q R_{2}\right)\left[J_{m}^{\prime}\left(k_{2} R_{1}\right) Y_{m}^{\prime}\left(k_{2} R_{2}\right)-Y_{m}^{\prime}\left(k_{2} R_{1}\right) J_{m}^{\prime}\left(k_{2} R_{2}\right)\right]\right\}=0 .
\end{array}
$$

\section{- Phonons of type 2}

$$
\begin{array}{r}
\varepsilon_{1 \infty} k_{1} J_{m}^{\prime}\left(k_{1} R_{1}\right)\left\{\varepsilon_{0} q K_{m}^{\prime}\left(q R_{2}\right)\left[I_{m}\left(k_{2} R_{1}\right) K_{m}\left(k_{2} R_{2}\right)-K_{m}\left(k_{2} R_{1}\right) I_{m}\left(k_{2} R_{2}\right)\right]\right. \\
\left.+\varepsilon_{2 \infty} k_{2} K_{m}\left(q R_{2}\right)\left[K_{m}\left(k_{2} R_{1}\right) I_{m}^{\prime}\left(k_{2} R_{2}\right)-I_{m}\left(k_{2} R_{1}\right) K_{m}^{\prime}\left(k_{2} R_{2}\right)\right]\right\} \\
+\varepsilon_{2 \infty} k_{2} J_{m}\left(k_{1} R_{1}\right)\left\{\varepsilon_{0} q K_{m}^{\prime}\left(q R_{2}\right)\left[K_{m}^{\prime}\left(k_{2} R_{1}\right) I_{m}\left(k_{2} R_{2}\right)-I_{m}^{\prime}\left(k_{2} R_{1}\right) K_{m}\left(k_{2} R_{2}\right)\right]\right. \\
\left.+\varepsilon_{2 \infty} k_{2} K_{m}\left(q R_{2}\right)\left[I_{m}^{\prime}\left(k_{2} R_{1}\right) K_{m}^{\prime}\left(k_{2} R_{2}\right)-K_{m}^{\prime}\left(k_{2} R_{1}\right) I_{m}^{\prime}\left(k_{2} R_{2}\right)\right]\right\}=0 .
\end{array}
$$

\section{- Phonons of type 3}

$$
\begin{array}{r}
\varepsilon_{1 \infty} k_{1} I_{m}^{\prime}\left(k_{1} R_{1}\right)\left\{\varepsilon_{0} q K_{m}^{\prime}\left(q R_{2}\right)\left[J_{m}\left(k_{2} R_{1}\right) Y_{m}\left(k_{2} R_{2}\right)-Y_{m}\left(k_{2} R_{1}\right) J_{m}\left(k_{2} R_{2}\right)\right]\right. \\
\left.+\varepsilon_{2 \infty} k_{2} K_{m}\left(q R_{2}\right)\left[Y_{m}\left(k_{2} R_{1}\right) J_{m}^{\prime}\left(k_{2} R_{2}\right)-J_{m}\left(k_{2} R_{1}\right) Y_{m}^{\prime}\left(k_{2} R_{2}\right)\right]\right\} \\
+\varepsilon_{2 \infty} k_{2} I_{m}\left(k_{1} R_{1}\right)\left\{\varepsilon_{0} q K_{m}^{\prime}\left(q R_{2}\right)\left[Y_{m}^{\prime}\left(k_{2} R_{1}\right) J_{m}\left(k_{2} R_{2}\right)-J_{m}^{\prime}\left(k_{2} R_{1}\right) Y_{m}\left(k_{2} R_{2}\right)\right]\right. \\
\left.+\varepsilon_{2 \infty} k_{2} K_{m}\left(q R_{2}\right)\left[J_{m}^{\prime}\left(k_{2} R_{1}\right) Y_{m}^{\prime}\left(k_{2} R_{2}\right)-Y_{m}^{\prime}\left(k_{2} R_{1}\right) J_{m}^{\prime}\left(k_{2} R_{2}\right)\right]\right\}=0 .
\end{array}
$$

\section{- Phonons of type 4}

$$
\begin{array}{r}
\varepsilon_{1 \infty} k_{1} I_{m}^{\prime}\left(k_{1} R_{1}\right)\left\{\varepsilon_{0} q K_{m}^{\prime}\left(q R_{2}\right)\left[I_{m}\left(k_{2} R_{1}\right) K_{m}\left(k_{2} R_{2}\right)-K_{m}\left(k_{2} R_{1}\right) I_{m}\left(k_{2} R_{2}\right)\right]\right. \\
\left.+\varepsilon_{2 \infty} k_{2} K_{m}\left(q R_{2}\right)\left[K_{m}\left(k_{2} R_{1}\right) I_{m}^{\prime}\left(k_{2} R_{2}\right)-I_{m}\left(k_{2} R_{1}\right) K_{m}^{\prime}\left(k_{2} R_{2}\right)\right]\right\} \\
+\varepsilon_{2 \infty} k_{2} J_{m}\left(k_{1} R_{1}\right)\left\{\varepsilon_{0} q K_{m}^{\prime}\left(q R_{2}\right)\left[K_{m}^{\prime}\left(k_{2} R_{1}\right) I_{m}\left(k_{2} R_{2}\right)-I_{m}^{\prime}\left(k_{2} R_{1}\right) K_{m}\left(k_{2} R_{2}\right)\right]\right. \\
\left.+\varepsilon_{2 \infty} k_{2} K_{m}\left(q R_{2}\right)\left[I_{m}^{\prime}\left(k_{2} R_{1}\right) K_{m}^{\prime}\left(k_{2} R_{2}\right)-K_{m}^{\prime}\left(k_{2} R_{1}\right) I_{m}^{\prime}\left(k_{2} R_{2}\right)\right]\right\}=0 .
\end{array}
$$

For quantum wires with an outer metallic shell the dispersion relations are somewhat simpler and they read 


\section{- Phonons of type 1}

$$
\begin{aligned}
& \varepsilon_{1 \infty} k_{1} J_{m}^{\prime}\left(k_{1} R_{1}\right)\left\{J_{m}\left(k_{2} R_{1}\right) Y_{m}\left(k_{2} R_{2}\right)-Y_{m}\left(k_{2} R_{1}\right) J_{m}\left(k_{2} R_{2}\right)\right\} \\
& +\varepsilon_{2 \infty} k_{2} J_{m}\left(k_{1} R_{1}\right)\left\{Y_{m}^{\prime}\left(k_{2} R_{1}\right) J_{m}\left(k_{2} R_{2}\right)-J_{m}^{\prime}\left(k_{2} R_{1}\right) Y_{m}\left(k_{2} R_{2}\right)\right\}=0 .
\end{aligned}
$$

- Phonons of type 2

$$
\begin{aligned}
& \varepsilon_{1 \infty} k_{1} J_{m}^{\prime}\left(k_{1} R_{1}\right)\left\{I_{m}\left(k_{2} R_{1}\right) K_{m}\left(k_{2} R_{2}\right)-K_{m}\left(k_{2} R_{1}\right) I_{m}\left(k_{2} R_{2}\right)\right\} \\
& +\varepsilon_{2 \infty} k_{2} J_{m}\left(k_{1} R_{1}\right)\left\{K_{m}^{\prime}\left(k_{2} R_{1}\right) I_{m}\left(k_{2} R_{2}\right)-I_{m}^{\prime}\left(k_{2} R_{1}\right) K_{m}\left(k_{2} R_{2}\right)\right\}=0 .
\end{aligned}
$$

- Phonons of type 3

$$
\begin{aligned}
& \varepsilon_{1 \infty} k_{1} I_{m}^{\prime}\left(k_{1} R_{1}\right)\left\{J_{m}\left(k_{2} R_{1}\right) Y_{m}\left(k_{2} R_{2}\right)-Y_{m}\left(k_{2} R_{1}\right) J_{m}\left(k_{2} R_{2}\right)\right\} \\
& +\varepsilon_{2 \infty} k_{2} I_{m}\left(k_{1} R_{1}\right)\left\{Y_{m}^{\prime}\left(k_{2} R_{1}\right) J_{m}\left(k_{2} R_{2}\right)-J_{m}^{\prime}\left(k_{2} R_{1}\right) Y_{m}\left(k_{2} R_{2}\right)\right\}=0 .
\end{aligned}
$$

\section{- Phonons of type 4}

$$
\begin{aligned}
& \varepsilon_{1 \infty} k_{1} I_{m}^{\prime}\left(k_{1} R_{1}\right)\left\{I_{m}\left(k_{2} R_{1}\right) K_{m}\left(k_{2} R_{2}\right)-K_{m}\left(k_{2} R_{1}\right) I_{m}\left(k_{2} R_{2}\right)\right\} \\
& +\varepsilon_{2 \infty} k_{2} J_{m}\left(k_{1} R_{1}\right)\left\{K_{m}^{\prime}\left(k_{2} R_{1}\right) I_{m}\left(k_{2} R_{2}\right)-I_{m}^{\prime}\left(k_{2} R_{1}\right) K_{m}\left(k_{2} R_{2}\right)\right\}=0 .
\end{aligned}
$$

Each equation from Eq. (13) to Eq. (20) for given $m$ and $q$ may have some roots for $\omega$ which can be ordered increasing gradually and indexed by integer $n$. Thus, index $v \equiv(t n)$ introduced in Eq. (6) indicates type $t$ of the phonon mode and the $n$th root of the corresponding dispersion equation. Different phonon branches of type $t$ are labeled by a couple of indexes $(m n)$.

For illustration we consider a $G a A s / A l_{x} G a_{1-x} A s$ quantum wire with $x=0.3$. The parameters for these chosen materials are: $\omega_{1 L}=36.2 \mathrm{meV}, \omega_{2 L}=34,5 \mathrm{meV}, \varepsilon_{1 \infty}=10.89, \varepsilon_{2 \infty}=$ $10.06, v_{1 L}=4.73 \mathrm{~m} / \mathrm{s}, v_{2 L}=4.97 \mathrm{~m} / \mathrm{s}, L=1 \mu \mathrm{m}$. Numerical calculations show that there do not exist phonons of type 3 and type 4 for quantum wires made of the two materials. Fig. 1 describes the $q$-dependence of frequencies for four lowest branches of phonons of type 1 and type 2 with azimuthal index $m=0$. The radii of the wire are taken as $R_{1}=3 \mathrm{~nm}, R_{2}=4 \mathrm{~nm}$.

It is seen that phonons of type 1 are of low frequencies while the eigenfrequencies of modes of type 2 are very near the limiting longitudinal optical frequency $\omega_{1 L}$. The modes of type 1 can be called the bulk-like ones since they propagate in both materials. Their related displacement fields given in Table 1 agree with those of LO confined bulk modes obtained by Enderlein in [24]. The difference is in the fact that the Enderlein's bulk modes are of two kinds: ones confined in material 1, the others in material 2. It comes from the assumption that the bulk dispersion curves of the materials do not overlap, i.e., the materials are of disparate mechanical properties so that oscillations in one material do not penetrate into the other. Meanwhile, oscillations under our consideration are supposed to be free of this assumption. This is the case of $G a A s / A l_{0.3} G a_{0.7} A s$ quantum wires.

It should be noted that the type-2-mode vibrational amplitudes consist of two components in the barrier material, one increasing and one decaying with increasing $r$ from the interface of the well material and the barrier one. If the barrier material fills up the space to infinity, the increasing component should be removed to ensure finite vibrational amplitudes. In this case the dispersion and the displacement field of the type-2 phonon modes are consistent with that of guided modes reported by Constantinou and Ridley in Ref. [30]. 

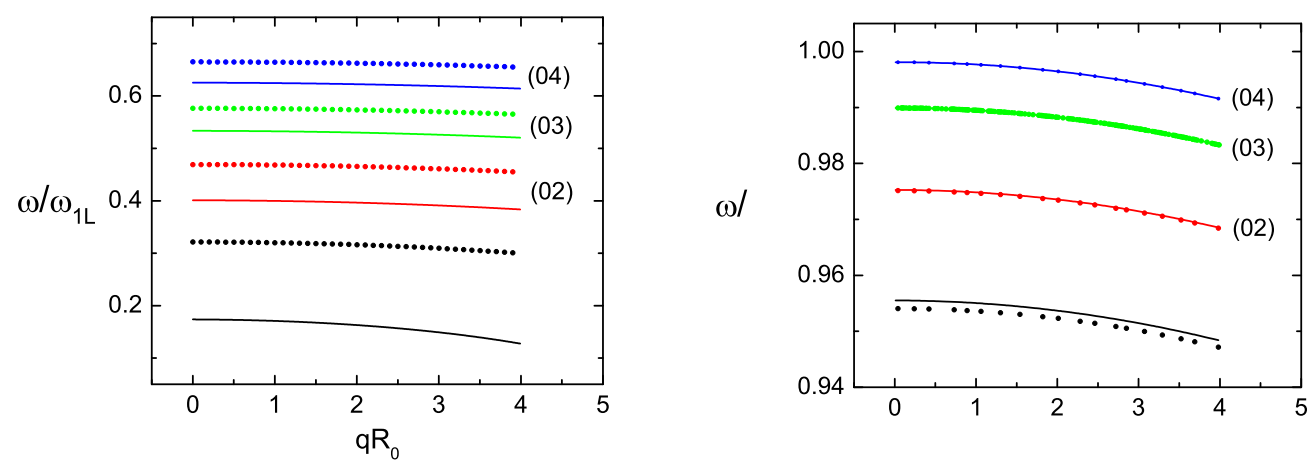

Fig. 1. Dispersion curves of the four lowest branches $(\mathrm{mn})$ of phonons of type 1 (left) and type 2 (right) with $m=0$ for a GaAs $/ A l G a A s$ quantum wire of $R_{1}=3 \mathrm{~nm}, R_{2}=4 \mathrm{~nm}$ without (solid line) or with (dotted line) a thin outer metallic shell.
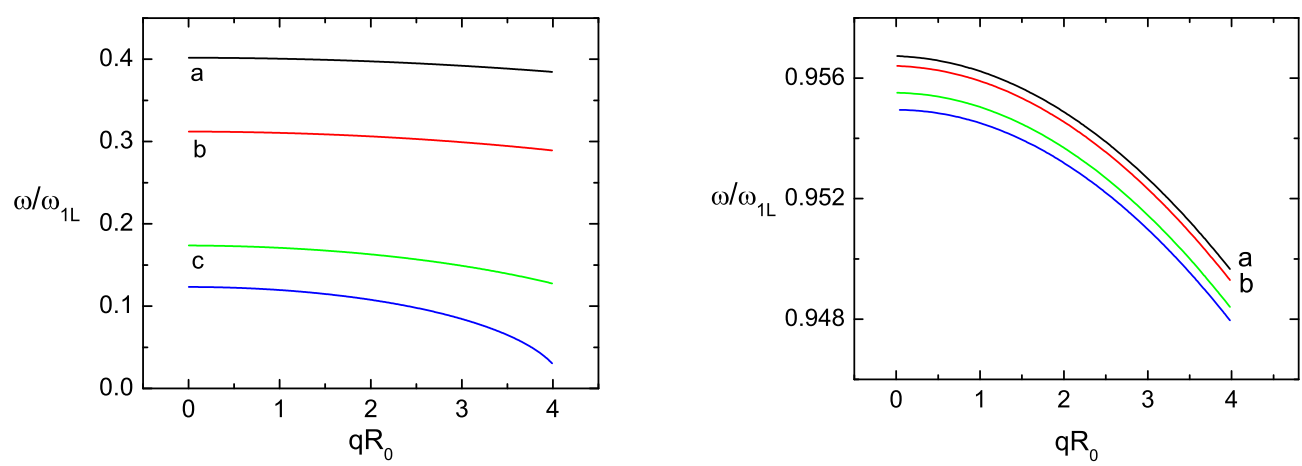

Fig. 2. Dispersion curves of phonons of type 1 (left) and type 2 (right) with $m=0, n=1$ for GaAs/AlGaAs quantum wires of $R_{1}=3 \mathrm{~nm}$ and various thickness of the barrier material $R_{2}-R_{1}$ : a) $0.02 \mathrm{~nm}$, b) $0.2 \mathrm{~nm}$, c) $1 \mathrm{~nm}$, and d) $30 \mathrm{~nm}$. The wires are without a thin outer metallic shell.

Fig. 1 depicts also the dispersion curves of phonon modes of both two types for quantum wires having an outer thin metallic shell. It is seen that, in comparison with case of wires without metallic shells, the eigenfrequencies have an upward shift for modes of type 1 and remain nearly unchanged for modes of type 2 .

In Fig. 2, we plot the wave-vector dispersions of type-1-phonon branch $(m=0, n=1)$ for quantum wires of the same $R_{1}$ but with various thickness of the barrier material.

As seen from the figure, the thicker barrier lowers the frequencies of phonons but does not affect their $q$-dependence. The change is more apparent for modes of type 1 .

\section{ELECTRON-LO-PHONON INTERACTION}

We introduce the electrostatic potential $\phi(\vec{x})$ which is associated with the electric field (12) via

$$
\vec{E}(\vec{x})=-\vec{\nabla} \phi(\vec{x})
$$


It follows from Eqs. (21), (12), and (7) that the potential related to the mode displacement $\vec{u}_{v m q}(\vec{x})$ can be expressed as

$$
\begin{aligned}
& \phi(\vec{x})=\eta(r) e^{i(m \varphi+q z)}, \\
& \eta(r)=4 \pi N_{j} e_{j}^{*} \psi_{v m q}(r) .
\end{aligned}
$$

Following the work by Ridley [39] the mode amplitude can be found by relating the total energy of the mode in the wire to that of an equivalent simple harmonic oscillator. The electron-LO-phonon interaction Hamiltonian is then given by the real part of the potential (22) multiplied by the charge of electron

$$
H=-\frac{e}{2}\left(\phi(\vec{x})+\phi^{*}(\vec{x})\right)
$$

Electrostatic potentials related to LO-modes are plotted in Fig. 3. The potentials for quantum wires with and without an outer metallic shell are shown in Fig. 4.
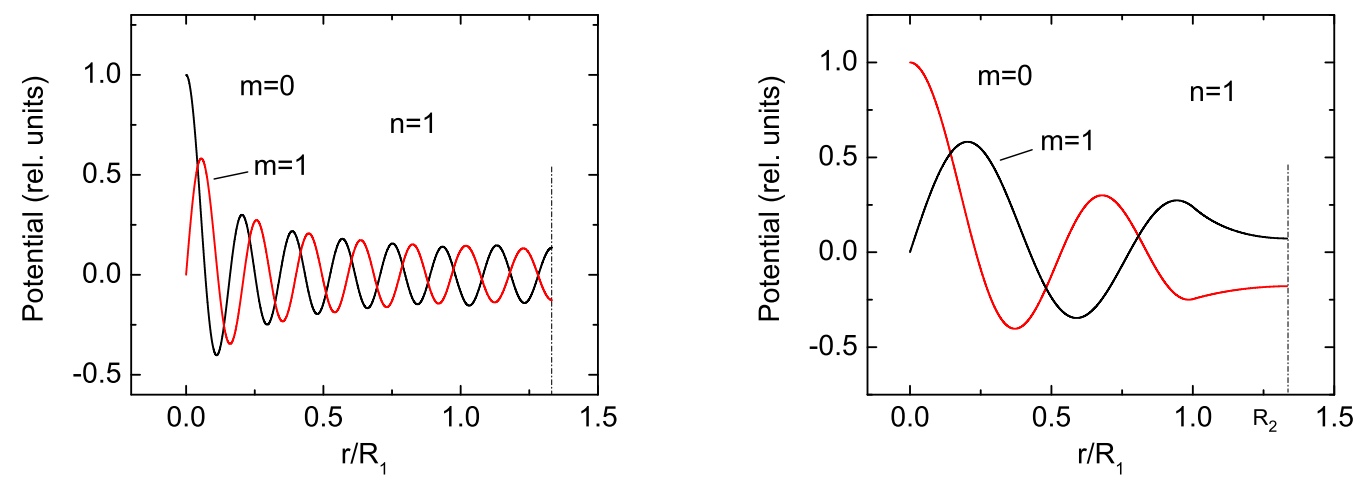

Fig. 3. Electrostatic potentials of phonon modes $(m n)$ of type 1 (left) and type 2 (right) for a $G a A s / A l G a A s$ quantum wire of $R_{1}=3 \mathrm{~nm}, R_{2}=4 \mathrm{~nm}$ without a thin outer metallic shell. The wave number $q$ is taken so as $q R_{1}=1$.
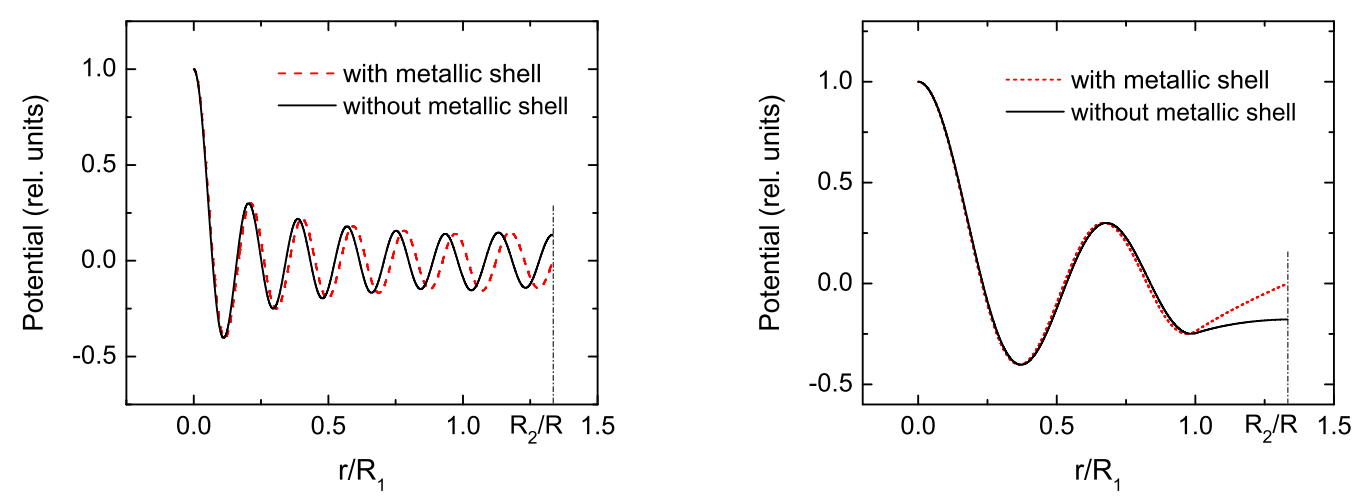

Fig. 4. Electrostatic potentials of phonon modes $(m=0, n=1)$ of type 1 (left) and type 2 (right) for a $G a A s / A l G a A s$ quantum wire without and with an outer thin metallic shell. The parameters are the same as in Fig. 3. 
It is seen that the metallic shell results in the change of the electrostatic potential which is more pronounced in the barrier region, especially for phonon modes of type 2. Therefore, one expects that the electron-LO-phonon interaction will be much affected by the presence of the outer metallic shell.

\section{CONCLUSION}

In this paper, under the dielectric continuum model, the longitudinal optical phonon modes of various types and their dispersion relations have been deduced for quasi-one-dimensional cylindrical quantum wires of core-shell structure. The electrostatic potential associated with the optical vibrations has also been derived to construct electron-LO-phonon interaction Hamiltonian. Numerical calculations performed for $G a A s / A l G a A s$ quantum wires showed that the mode dispersion and the related potential are modified by variation of the shell thickness and additionally by the presence of an outer metallic shell. It leads to the change in electron interactions with LO-phonons in quantum wires. The effects of finite thickness of the barrier material on electron-phonon scattering and phonon-assisted electron mobility in core-shell quantum wires are of interest and claim separate papers.

\section{REFERENCES}

[1] X. Duan, Y. Huang, and C. M. Lieber, Nano Letters 2 (5) (2002) 487-490.

[2] Y. Cui and C. M. Lieber, Science 291 (2001) 851-853.

[3] Y. Huang, X. Duan, Y. Cui, and C. M. Lieber, Nano Letters 2 (2) (2002) 101-104.

[4] Y. Cui, Q. Wei, H. Park, and C. M. Lieber, Science 293 (5533) (2001) 1289-1292.

[5] K. Karlsson, H. Weman, M.-A. Dupertuis, K. Leifer, A. Rudra, and E. Kapon, Physical Review B 70 (4) (2004) 045302.

[6] C. Eugster, J. Del Alamo, M. Rooks, and M. Melloch, Applied physics letters 64 (23) (1994) 3157-3159.

[7] S. Fischer, G. Apetrii, U. Kunze, D. Schuh, and G. Abstreiter, Nature Physics 2 (2) (2006) 91-96.

[8] C. H. Bennett and D. P. DiVincenzo, Nature 404 (6775) (2000) 247-255.

[9] H. J. Fan, P. Werner, and M. Zacharias, small 2 (6) (2006) 700-717.

[10] J. Leburton, Journal of applied physics 56 (10) (1984) 2850-2855.

[11] N. Constantinou and B. Ridley, Journal of Physics: Condensed Matter 1 (12) (1989) 2283.

[12] L. Wendler and R. Kugler, Journal of Physics: Condensed Matter 6 (39) (1994) 7857.

[13] C. Bennett, N. Constantinou, and B. Tanatar, Journal of Physics: Condensed Matter 7 (48) (1995) L669.

[14] N. Mansour, Y. M. Sirenko, K. Kim, M. Littlejohn, J. Wang, and J. Leburton, Applied physics letters 67 (23) (1995) 3480-3482.

[15] L. C. Fai, V. Teboul, A. Monteil, S. Maabou, and I. Nsangou, Condensed Matter Physics 8 (3) (2005) 639.

[16] G. Fasol, Phys. Rev. B 38 (1988) 6056.

[17] M. Watt, C. S. Torres, H. Arnot, and S. Beaumont, Semiconductor Science and Technology 5 (4) (1990) 285.

[18] K. Adu, Q. Xiong, H. Gutierrez, G. Chen, and P. Eklund, Applied Physics A 85 (3) (2006) 287-297.

[19] D. Spirkoska, G. Abstreiter, and A. F. i Morral, Nanotechnology 19 (43) (2008) 435704.

[20] S.-F. Ren and Y.-C. Chang, Physical Review B 43 (14) (1991) 11857.

[21] B.-f. Zhu, Semiconductor Science and Technology 7 (3B) (1992) B88.

[22] M. A. Stroscio, K. Kim, M. A. Littlejohn, and H. Chuang, Physical Review B 42 (2) (1990) 1488.

[23] P. Knipp and T. Reinecke, Physical Review B 45 (16) (1992) 9091.

[24] R. Enderlein, Physical Review B 47 (4) (1993) 2162.

[25] C. Bennett and B. Tanatar, Physical Review B 55 (11) (1997) 7165.

[26] H.-J. Xie, C.-Y. Chen, and B.-K. Ma, Physical Review B 61 (7) (2000) 4827.

[27] Z. Li, Communications in Theoretical Physics 42 (3) (2004) 459.

[28] A. L. Vartanian, International Journal of Modern Physics B 20 (21) (2006) 3015-3025. 
OPTICAL PHONON MODES AND ELECTRON-OPTICAL PHONON INTERACTION ...

[29] Z.-W. Zuo and H.-J. Xie, Journal of Physics: Condensed Matter 22 (2) (2010) 025403.

[30] N. Constantinou and B. Ridley, Physical Review B 41 (15) (1990) 10627.

[31] M. A. Stroscio and M. Dutta, Phonons in nanostructures, Cambridge University Press, 2001.

[32] B. K. Ridley, Electrons and phonons in semiconductor multilayers, vol. 5, Cambridge University Press, 2009.

[33] B. Ridley, Physical Review B 47 (8) (1993) 4592.

[34] X. Wang and X. Lei, Solid state communications 91 (7) (1994) 513-517.

[35] F. Comas, A. Cantarero, C. Trallero-Giner, and M. Moshinsky, Journal of Physics: Condensed Matter 7 (9) (1995) 1789.

[36] V. Stavrou, C. Bennett, O. Al-Dossary, and M. Babiker, Physical Review B 63 (20) (2001) 205304.

[37] F. Comas, I. Camps, G. Marques, and N. Studart, Semiconductor science and technology 22 (3) (2007) 229.

[38] I. Gradshteyn, Series, and Products, Alan Jeffrey and Daniel Zwillinger (eds.), Seventh edition (Feb 2007) 885 (2007) .

[39] B. Ridley, Physical Review B 39 (8) (1989) 5282. 